\title{
Apprendre à lire en FLE : état des lieux dans une province du Maroc
}

\author{
${ }^{1}$ Ismail KHATTALA \& ${ }^{2}$ Rachida BOUALI \\ 1,2 Faculté des lettres et sciences humaines, Université Mohammed Premier Oujda - Maroc
}

Reçu le 24 mai 2019 | Accepté le 30 juin 2019

\begin{abstract}
RÉSUMÉ. Cet article rend compte des résultats d'une enquête que nous avons menée par le biais $d^{\prime}$ 'un questionnaire auprès d'enseignants de la langue française du primaire. L'objectif était de dresser l'état des lieux de l'enseignement/apprentissage de la lecture orale et d'identifier les difficultés qui entravent la maîtrise de cette compétence à partir du point de vue des praticiens. Cette enquête qui a eu lieu au sein d'écoles primaires publiques d'une province de l'oriental au Maroc nous a permis $\mathrm{d}^{\prime}$ explorer la pratique enseignante de la lecture et d'évaluer les connaissances des enseignants relatives à la conscience phonologique qui constitue une composante fondamentale de cet apprentissage et l'un des meilleurs prédicteurs de sa future réussite. Les données collectées indiquent que les enseignants sont insatisfaits des performances de leurs élèves en lecture et que ces derniers souffrent d'un déficit grave en déchiffrage. De plus, nous avons pu déduire que les stratégies mises en œuvre par nos informateurs pour remédier à cette déficience ne sont que peu efficaces. Les connaissances de ces praticiens relatives au rôle important que joue le développement de la conscience phonologique lors de l'apprentissage de la lecture sont très limitées d'où la nécessité d'assurer des formations supplémentaires à ce propos.
\end{abstract}

Mots-clés : conscience phonologique, difficultés de lecture, identification des mots écrits, lecture, Maroc

\begin{abstract}
This article reports on the results of a survey that we conducted through a questionnaire with teachers of the French primary language. The aim was to take stock of the teaching / learning of oral reading and to identify the difficulties that hinder the mastery of this competence from the point of view of practitioners. This survey, which took place in public primary schools in an Eastern province in Morroco, allowed us to explore the teaching practice of reading and assess teachers' knowledge of phonological awareness as a component of this learning and one of the best predictors of future success. The data collected indicates that teachers are dissatisfied with the performance of their students in reading and that they suffer from a serious deficit in deciphering. In addition, we have been able to deduce that the strategies implemented by our informants to remedy this deficiency are only slightly effective. The knowledge of these practitioners about the important role played by the development of phonological awareness when learning to read is very limited, hence the need for additional training in this area.
\end{abstract}

Keywords: phonological awareness, reading difficulties, identification of written words, reading, Morocco

国 auteur correspondant : ismail.khattala@gmail.com

Pour citer cet article (Style APA) : Khattala \& Bouali. (2019). Apprendre à lire en FLE : état des lieux dans une province du Maroc. Francisola: Revue Indonésienne de la langue et la littérature françaises, 4(1), 45-54 . doi: 10.17509/francisola.v4i1.20341. 


\section{INTRODUCTION}

$\mathrm{Au}$ Maroc, apprendre à lire en langue française revêt une importance cruciale, pourtant, les différentes évaluations qui ont porté sur les acquis scolaires durant la dernière décennie dévoilent une déficience alarmante en termes d'apprentissage de cette langue sachant qu'elle bénéficie d'un statut très privilégié comme l'explique Toumi:

Actuellement le français jouit encore d'une importance particulière dans la société marocaine :

- Langue d'enseignement des sciences à l'université.

- Langue de promotion sociale.

- Clef de la réussite aux examens.

- Moyen d'accès au marché du travail.

- Moyen de fonctionnement de l'administration dans certains ministères, ainsi que dans

- plusieurs secteurs commerciaux, bancaires et en particulier dans le secteur privé.

- Moyen d'expression culturelle, médiatique et artistique. (2016, p.92)

De plus, la langue française est en train de devenir selon les réformes en cours, la vision stratégique (2015-2030), une langue d'enseignement de certaines disciplines scientifiques dès le primaire.

Tout cela fait de l'apprentissage de la lecture dans cette langue une nécessité indispensable à la réussite scolaire et professionnelle de l'élève marocain. Pourtant, cet apprentissage, au Maroc comme en France, devient de plus en plus problématique et menace sérieusement la réussite de l'acquisition de cette langue. En effet, la France s'est située en queue de classement des pays européen en lecture selon le dernier rapport du PIRLS 2016 (Programme international de recherche en lecture scolaire). Concernant le Maroc, un rapport récent du Conseil supérieur de l'éducation, de la formation et de la recherche scientifique marocain (PNEA 2016) a révélé une insuffisante maitrise de la lecture en français par les élèves du tronc commun (première année du lycée).

C'est dans cette perspective que s'inscrit l'étude dont nous rapportons les résultats dans le présent article. Il s'agit d'une étude exploratoire que nous avons menée au biais d'un questionnaire distribué aux enseignants du cycle primaire d'une province de l'Oriental du Maroc dans l'objectif de dresser l'état des lieux de cet enseignement et d'identifier les difficultés qui entravent la maîtrise de la lecture en langue française du point de vue des praticiens.

Pour une meilleure compréhension des items de ce questionnaire ainsi que de la logique qui a présidé à son élaboration, Nous allons en ce qui suit faire état des connaissances théoriques dont nous disposons actuellement qui portent sur le fonctionnement cognitif de l'apprentissage de la lecture ainsi que sur l'une de ses principales composantes : la conscience phonologique.

À ce propos, le dictionnaire pratique de didactique du FLE définit l'acte de lire comme suit:

En linguistique, lire, c'est : savoir utiliser le code écrit d'une langue, savoir décoder un message écrit qu'un scripteur a préalablement encodé conformément aux normes de la langue utilisée et du type du message réalisé. En didactique des langues, lire, c'est: s'approprier le sens d'un message. Pour cela, le lecteur doit justifier à la fois d'un savoir (représenté par l'ensemble de ses connaissances linguistiques et extra-linguistiques) et d'un savoir-faire (constitué de stratégies permettant la réalisation des trois opérations fondamentales de l'acte de lire : anticiper, identifier, vérifier). (Robert, 2002, p.98).

De même, l'Observatoire National de la Lecture (ONL) (2005, p.9) précise que:

Lire, c'est extraire d'une représentation graphique du langage la prononciation et la signification qui lui correspondent. On lit pour comprendre. La dimension de compréhension n'est toutefois pas spécifique à la lecture, elle préexiste à l'apprentissage de la lecture et s'exerce à la fois au cours et en dehors de cet apprentissage. Le problème auquel se trouve confronté l'enfant qui entre à l'école élémentaire est d'apprendre à identifier les mots écrits et de mettre en œuvre l'activité de compréhension à 
partir de cette identification.

Ces différentes définitions nous mènent à déduire que la compréhension des messages encodés par écrit demeure la finalité de la lecture et qu'il s'agit d'une compétence complexe qui fait appel, au moins, à deux capacités fondamentales : l'identification des mots et la compréhension à l'oral. À cet égard, Bentolila nous explique dans l'introduction d'une autre publication de l'Observatoire National de la Lecture (ONL), (1998, p.21) que :

En règle générale, les recherches font apparaitre que les jeunes adultes en difficulté de lecture ont une capacité d'identification des mots insuffisante [...], il importe donc que l'on veille tout particulièrement à ce que tous les élèves apprennent à identifier les mots avec efficacité, c'est-à-dire en alliant rapidité et précision.

Dans le même sens, Gombert (1990, p.209) ajoute que :

La reconnaissance des mots conditionne toute l'activité de lecture car elle est un préalable à la compréhension. De plus, il ne suffit pas qu'elle soit correctement opérée. Il faut également que son coût cognitif soit suffisamment peu élevé pour que le lecteur puisse allouer de l'attention à des activités de compréhension de plus haut niveau.

Par conséquent, l'identification des mots écrits, avec aisance et précision, constitue un apprentissage de base qui doit être suffisamment maitrisé dès le début de l'enseignement de la lecture en n'importe quelle langue alphabétique et quel que soit le contexte de son apprentissage. À ce propos, des recherches empiriques qui ont porté sur cette compétence ont révélé la coexistence de deux voies de la lecture experte qui se complètent et qui fonctionnement comme suit :

La plupart des modèles de la lecture postulent que deux voies de traitement de l'information coexistent et se complètent. Lorsque nous lisons des mots rares, nouveaux, à l'orthographe régulière, voire des néologismes inventés de toutes pièces, notre lecture passe par une voie phonologique qui décrypte les lettres, en déduit une prononciation possible, puis tente d'accéder au sens.
Inversement, lorsque nous sommes confrontés à des mots fréquents ou irréguliers, notre lecture emprunte une voie directe, qui récupère d'abord le mot et son sens, puis utilise ces informations pour en recouvrer la prononciation. (Dehaene, 2007, p.68).

On n'en déduit donc que le lecteur dispose principalement de deux stratégies pour identifier un mot écrit selon la nature et le niveau de sa familiarité avec ce dernier :

- Une voie dite d'adressage (directe ou orthographique) pour reconnaitre d'une façon globale des mots qu'il a déjà rencontrés et mémorisés : «Dans ce cas, aucune information phonologique n'intervient dans le processus d'identification des mots, la prononciation du mot s'effectue sur la base d'un code phonologique récupéré directement dans le lexique mental» (Ecalle et Magnan, 2002, p.26)

- Une voix d'assemblage (indirecte ou phonologique) qui se caractérise par le recours aux conversions graphophonologiques afin d'identifier les mots nouveaux (ou pseudomots) dont la forme orthographique lui est inconnue.

Concernant l'installation de ces deux voies de lecture, Gillig (2009, p.10) précise que «plus le lecteur débutant devient habile, moins il aura à utiliser l'assemblage, puisqu'il active toujours davantage l'identification des mots déjà vus par reconnaissance directe ». Apprendre à lire revient donc à développer ces deux voies et à automatiser l'identification des mots en privilégiant la voie directe qui permet de libérer les ressources cognitives importantes mobilisées lors du déchiffrage, ce qui est primordial à la compréhension.

À ce propos, de nombreuses études ont démontré que le développement précoce de la conscience phonologique aide les enfants à comprendre le principe alphabétique et qu'il est l'un des meilleurs prédicteurs de sa réussite. Dehaene et al., (2011, p.26) expliquent à cet égard que l'apprentissage de la lecture «consiste à prendre conscience des structures du langage oral, afin de les mettre en rapport avec le 
code visuel des lettres ». Briquet-Duhazé (2013, p.9) stipule que la conscience phonologique est «une compétence métalinguistique qui consiste à opérer des tâches délibérées sur les mots à l'oral ». Elle assure que « la recherche scientifique montre de manière consensuelle que l'enfant doit savoir analyser intentionnellement le langage oral afin de réussir à apprendre à lire ». (2013, p.9). Il s'agit donc d'amener l'apprenti lecteur à comprendre que la langue orale est constituée d'éléments plus petits à savoir les mots, les syllabes et les phonèmes et que ces éléments ont des correspondants à l'écrit et à les manipuler d'une manière explicite et intentionnelle dans des tâches d'identification, de catégorisation, de substitution, de suppression et d'ajout.

En ce qui concerne le rapport entre la conscience phonologique et la lecture, il existe actuellement un large consensus qu'il s'agit d'une relation bidirectionnelle dans la mesure où la conscience phonologique est en même temps un prérequis et une résultante de l'apprentissage de la lecture et plus précisément du déchiffrage comme l'expliquent Ecalle et Magnan (2002, p.86) : «la compétence phonologique apparait comme bi-directionnelle, à la fois cause et conséquence de la langue écrite et constitue un bon prédicteur de la réussite en lecture ».

L'importance de ces habiletés métaphonologiques a été mise en évidence par une méta-analyse récente de MelbyLervag, Lyster et Hulme (2012) qui ont pu déduire à partir des résultats de 235 études que la conscience phonémique (relative à la manipulation des phonèmes) peut renseigner les différences individuelles au cours de l'apprentissage de la lecture. En revanche, d'autres études ont pu démontrer que les performances des élèves dans des tâches de conscience phonologique s'améliorent au fur et à mesure qu'il progresse dans leur apprentissage de la lecture (Algéria et Morais, 1979 cité dans Ecalle, J. et Magnan, A., 2015.).

\section{MÉTHODE}

\subsection{Présentation du questionnaire et des objectifs de l'étude}

La première partie de ce questionnaire était dédiée à l'enseignement apprentissage de la lecture tandis que la deuxième a porté sur la conscience phonologique en relation avec les différentes activités pratiquées en classe de français. Les principaux objectifs qui ont motivé cette investigation étaient les suivants :

- Situer le niveau de nos élèves en lecture oralisée et identifier les difficultés qui entravent cet apprentissage.

- Explorer les pratiques enseignantes de remédiation ainsi que celles de développement de la conscience phonologique.

Pour l'identification des difficultés de lecture, nous en avons proposé une série allant de la connaissance des noms des lettres jusqu'à la compréhension des textes lus en des difficultés relatives à la prononciation. Pour ces questions, nous avons laissé de l'espace pour que les informateurs puissent nous renseigner sur d'autres éventuelles difficultés que nous n'avons pas suggérées. La partie du questionnaire qui a porté sur la conscience phonologique avait pour objectif principal de rendre compte des connaissances des praticiens à ce propos. Les items proposés étaient axés autour de quelques capacités de la conscience phonologique qui sont en relation avec l'apprentissage de la lecture ainsi que sur le soutien des élèves en difficultés de déchiffrage.

\subsection{Contexte d'étude et population cible \\ Cette investigation s'est déroulée en} fin de l'année scolaire 2015-2016 (mai 2016) dans des écoles primaires publiques de la direction provinciale de Taourirt (Académie régionale de l'Oriental). 76 enseignants du français y ont participé (soit environ le $1 / 4 \mathrm{du}$ nombre total des enseignants du français de notre contexte d'étude). Après avoir obtenu les autorisations nécessaires, nous avons visité les établissements scolaires visés par l'étude pour distribuer les questionnaires. Au cours de ces visites, nous avons expliqué à tous les informateurs les objectifs de notre recherche ainsi que les items du 
questionnaire. Nous leur avons accordé un délai de deux semaines pour le remplir afin de leur donner suffisamment de temps pour répondre avec pertinence et fiabilité. Pour l'échantillonnage, nous avons essayé de couvrir les différents milieux socioculturels des élèves en choisissant des établissements dispersés aux quatre coins des deux principales villes de la province ainsi que les écoles rurales qui se trouvent à leurs alentours.

\section{RÉSULTATS ET DISCUSSION}

Le traitement et l'analyse des résultats recueillis nous ont fourni les résultats suivants :

\subsection{Caractéristiques des informateurs et du contexte d'étude}

- Nombre de participants : 76 enseignants ont répondu à notre questionnaire dont $61,80 \%$ étaient des hommes et $38,20 \%$ des femmes.

- Âges : 6,6\% étaient âgés de moins de 30 ans, 35,5\% avaient entre 30 et 40 ans et $57,90 \%$ étaient âgés de plus de 40 ans. $75 \%$ de ces enseignants avaient plus de dix ans d'expérience dont $46 \%$ en avaient plus de 20ans.

- Répartition des informateurs selon le milieu d'exercice : $72,40 \%$ des informateurs travaillaient dans des écoles urbaines et 27,60\% dans le milieu rural.

Les caractéristiques que nous venons de présenter sont, en fait, très bénéfiques pour notre investigation puisque l'expérience professionnelle assez importante des informateurs est susceptible de garantir une étude plus pertinente de la problématique.

Lors de cette enquête, nous avons écarté les enseignants des deux premières années du primaire puisque l'enseignement apprentissage de la lecture en langue française commence dans les établissements publiques en troisième année et se poursuit jusqu'à la fin de ce cycle (sixième année).

\subsection{L'enseignement apprentissage de la lecture : état des lieux.}

L'objectif des questions proposées dans cette partie de notre questionnaire était de rendre compte de la pratique effective de l'enseignement apprentissage de la lecture, de son efficacité ainsi que de ses difficultés.

- Degré de satisfaction des praticiens des performances de leurs élèves en lecture oralisée: Les résultats recueillis indiquent que seulement $17,10 \%$ des informateurs sont satisfaits du niveau de leurs élèves en lecture oralisée, au moment où $61,80 \%$ sont insatisfaits et $21,10 \%$ ont manifesté leur inquiétude. De plus, $54 \%$ de ces informateurs ont déclaré avoir un taux d'élèves en difficultés de lecture situé entre $40 \%$ et $80 \%$, ce qui est très inquiétant vu l'importance de cette compétence qui est indispensable à la réussite de l'apprentissage des autres disciplines linguistiques enseignées.

Pour déterminer les causes de cette déficience qui s'annonce pessimiste, mais non surprenante, nous avions prévu une série de questions pour faire le diagnostic de cette situation :

- Le temps moyen accordé en classe à chaque élève pour lire oralement : A ce propos, 63\% des enseignants ont affirmé que le temps qu'ils accordaient à chaque élève pour lire oralement en classe ne dépassait pas, en moyenne, cinq minutes par semaine. Ajoutons à cela que la répartition de ce temps est loin d'être équitable. Cette durée diffère considérablement d'une classe à l'autre et selon les élèves, elle se trouve influencée par plusieurs facteurs dont nous citons quelques-uns :

1. L'effectif de la classe qui est souvent trop élevé surtout dans les villes, il dépassait (à l'époque de l'enquête) en moyenne 35 élèves par classe surtout pour les niveaux supérieurs (5AEP et 6AEP) qui connaissent des taux de redoublement plus importants.

2. En milieu rural, l'effectif est beaucoup moins élevé, mais une autre contrainte réduit le temps accordé à l'élève pour lire en classe : c'est le problème des classes 
combinées. Les enseignants dans ce contexte prennent en charge aux moins deux niveaux dans le même groupe classe, il peut atteindre jusqu'à quatre niveaux différents voire même six niveaux dans certains cas.

3. Facteurs d'ordre pédagogique: ils concernent surtout la gestion du temps par certains enseignants qui ne le répartissent pas d'une manière équitable entre les élèves : les bons lecteurs sont souvent plus favorisés que ceux en difficultés, en plus, le temps consacré aux lectures individuelles s'épuise rapidement afin de pouvoir passer aux activités dédiées à la compréhension et au réinvestissement des textes lus.

4. Facteurs d'ordre psychologique et motivationnel : les élèves ayant des problèmes de décodage évitent de lire à haute voix en classe par peur de se faire ridiculiser.

\subsection{Les difficultés de lecture constatées par les enseignants. \\ En termes de cette question, nous} avons demandé aux enseignants de nous renseigner sur les difficultés de lecture les plus récurrentes dans leurs classes et $\mathrm{d}$ 'indiquer dans une liste que nous avons proposée celles qu'ils considèrent problématiques pour leurs élèves et d'en ajouter d'autres que nous n'avions peut être pas prévues.

$\mathrm{D}^{\prime}$ après les réponses fournies à cette question, nous avons constaté que les difficultés relatives à la compréhension de l'écrit sont omniprésentes puisque $69,70 \%$ des informateurs ont déclaré en avoir dans leurs classes, suivies, dans un ordre décroissant d'importance : des problèmes liés à la prononciation, au déchiffrage des mots irréguliers et à la manipulation des syllabes et des phonèmes qui composent les mots. En revanche, Nous avons pu déduire que la connaissance des noms des lettres est la moins problématique dans notre contexte d'étude, surtout pour les élèves des niveaux supérieurs.

De plus, les réponses recueillies indiquent que les correspondances graphophonémiques ainsi que certaines habiletés métaphonologiques (la fusion des lettres pour former les syllabes ainsi que la manipulation de celles-ci ) ne sont pas suffisamment maitrisées pour permettre aux élèves de lire d'une manière correcte et fluide et nécessitent donc plus d'attention de la part des enseignants. La figure (1) expose les réponses fournies à cette question.

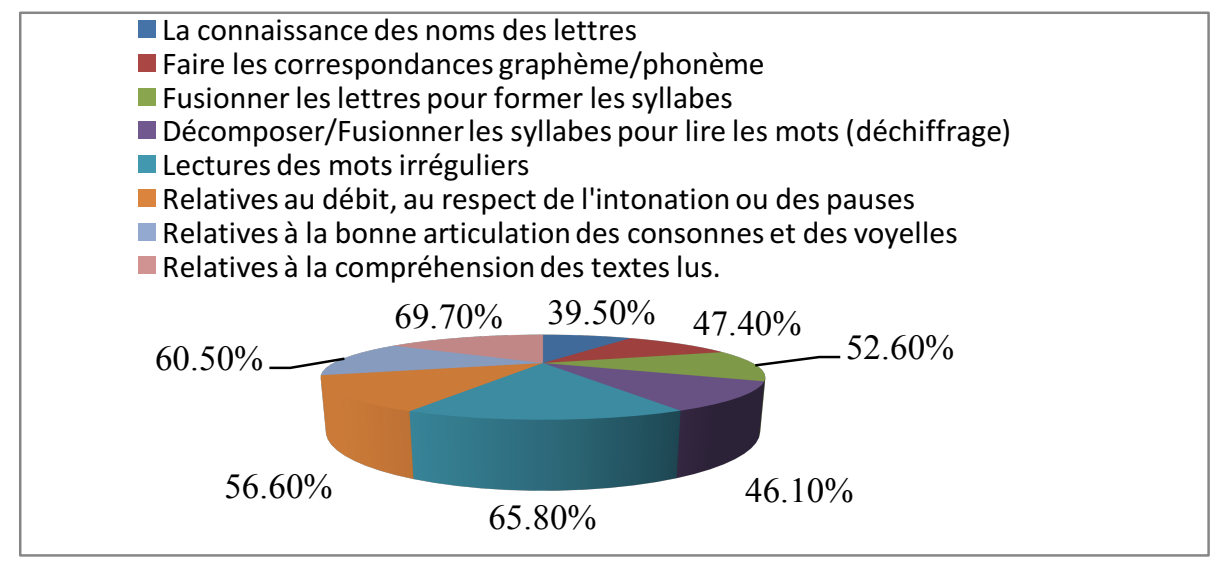

Figure 1: Taux de récurrences des difficultés en lecture oralisée 


\subsection{Stratégies de remédiation adoptées par les enseignants \\ Pour enchainer avec les questions} de la rubrique précédente, Nous avons interrogé ensuite les enseignants à propos des stratégies de remédiation qu'ils mettent en œuvre au profit de leurs élèves pour les aider à surmonter les difficultés de lecture qu'ils éprouvent. La question était accompagnée par quelques propositions que nous avons fournies mais nos informateurs avaient la possibilité de nous renseigner sur d'éventuelles activités de remédiation autres que celles que nous avons proposées. Les réponses fournies (figure 2) indiquent que les efforts des enseignants penchent surtout sur la maitrise du décodage graphophonologique et l'enrichissement du répertoire lexical des élèves dans l'objectif d'améliorer leurs performances en compréhension.

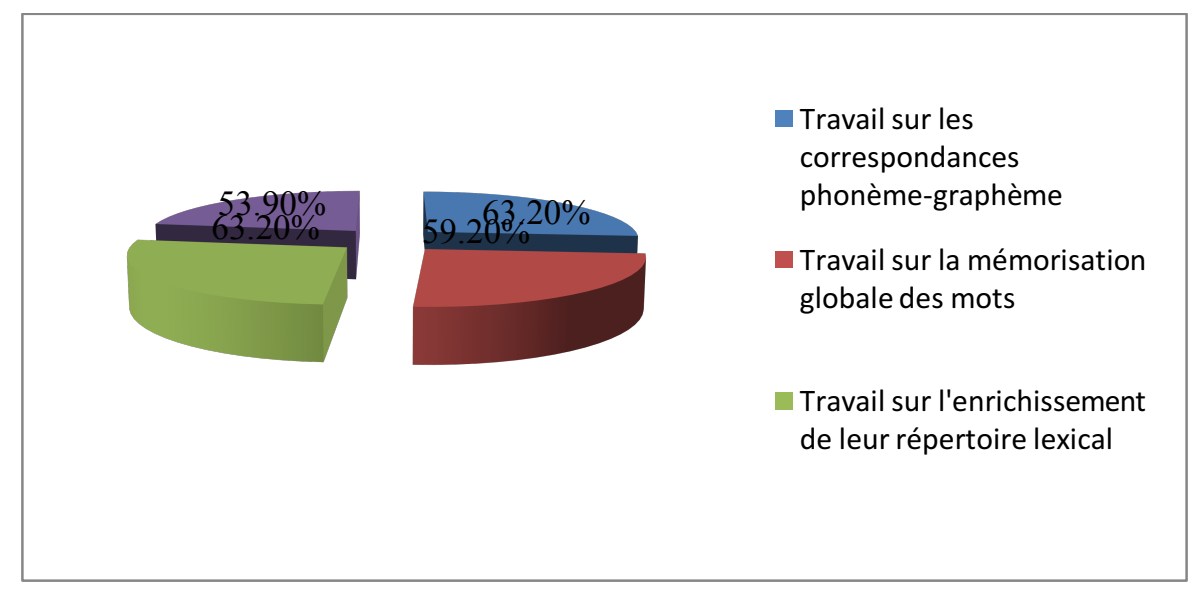

Figure 2: Fréquences des activités de remédiation mises en œuvre

Nous avons évité d'insérer la conscience phonologique parmi les stratégies d'aide que nous avons proposées dans l'espoir de la retrouver dans les solutions indiquées par les enseignants mais aucun d'eux n'a fait allusion à cette compétence malgré son importance.

\subsection{Activités orales en relation avec l'apprentissage de la lecture}

Par cette question, nous avons cherché à connaitre les activités orales pratiquées en classe qui visent à aider les élèves à améliorer leurs performances en lecture sachant que cette compétence, comme nous l'avons précisé au début de cet article, fait appel à plusieurs connaissances et capacités qui relèvent de l'oral comme de l'écrit. D'après les réponses fournies par les enseignants (figure 3), nous constatons que ces activités portent, pour la plupart, sur la correction phonétique et le travail sur le lexique, celles de développement de la conscience phonologique à travers les exercices de manipulation des syllabes et des phonèmes sont beaucoup moins importantes surtout en fin du primaire. Cette attitude est justifié par les enseignants des niveaux supérieurs par le fait que ces habiletés se développement, selon les programmes en vigueur, en 3ème année primaire. A partir de ces réponses, nous pouvons déduire que l'apport du développement de la conscience phonologique à l'apprentissage de la lecture et au soutien des élèves en difficulté est mal connu et peu exploité par ces enseignants. A cet égard, Briquet-Duhazé (2013, p.102) a constaté suite à une évaluation de la 
conscience phonologique d'élèves les bons lecteurs avaient obtenus de français scolarisés en fin du cycle 3, que meilleurs résultats que les faibles.

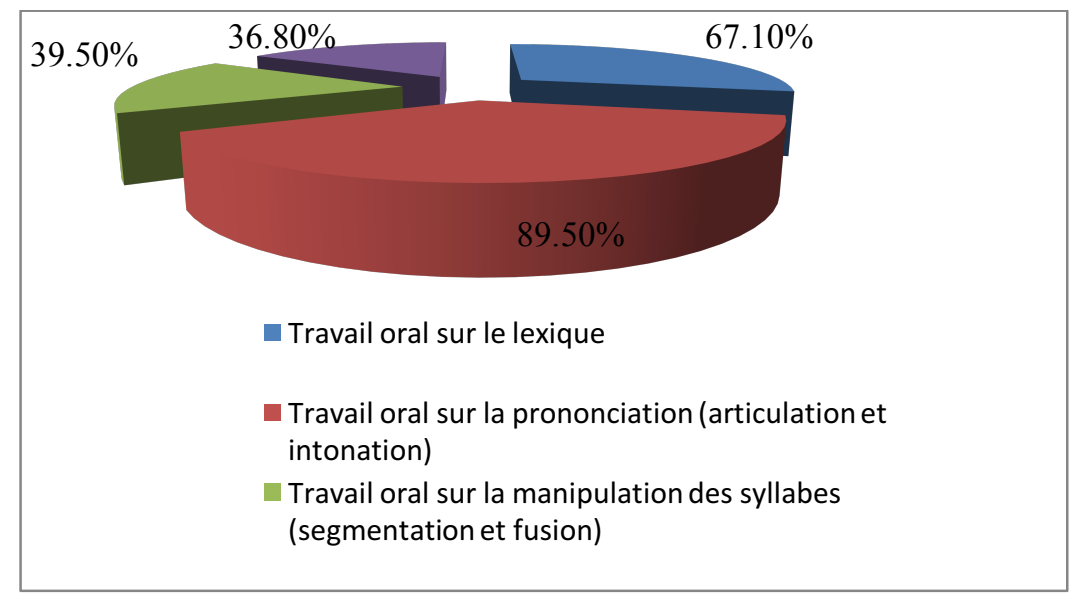

Figure 3: Activités orales pratiquées en relation avec l'apprentissage de la lecture

3.6. Enseignement de la lecture et conscience phonologique (C.P)

Il s'agit dans cette partie du questionnaire d'interroger la pratique enseignante relative au développement de la conscience phonologique. A ce propos, $85 \%$ des informateurs ont déclaré que leurs connaissances étaient insuffisantes et ils qu'ils avaient besoin de formations à ce sujet. Pourtant, et même si la majorité de ces enseignants n'avaient jamais entendu parler de la conscience phonologique auparavant, presque $60 \%$ d'entre eux pratiquaient, sans le savoir, des activités en relation avec son développement, et $19,70 \%$ ont déclaré qu'ils le faisaient souvent (figure 4).

En parfaite cohérence avec les constats que nous avons faits précédemment, le développement de la conscience phonologique se fait exclusivement en troisième année du primaire lors de l'enseignement du déchiffrage, et il devient de moins en moins pratiqué ensuite même en guise de remédiation aux lacunes d'apprentissage de la lecture accumulées par les élèves.

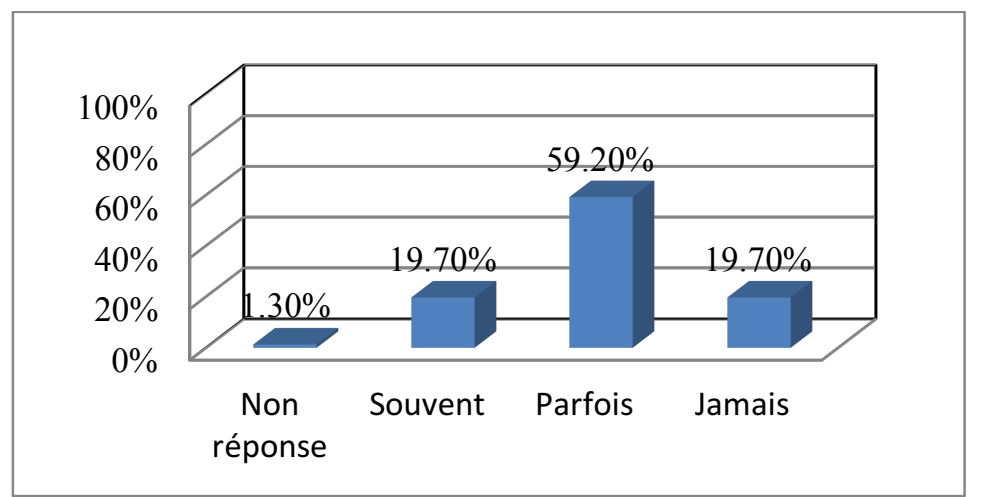

Figure 4: Fréquences des activités de développement de la conscience phonologique pratiquées 
Les activités les plus pratiquées sont celles qui portent sur la décomposition des mots en syllabes et le repérage des syllabes. Nous retrouvons ensuite les activités de discrimination syllabique suivies de celle de repérage et de discrimination des phonèmes. Nous remarquons aussi sur la figure 5 que les activités de manipulation phonémique sont moins pratiquées que celles qui portent sur les syllabes.

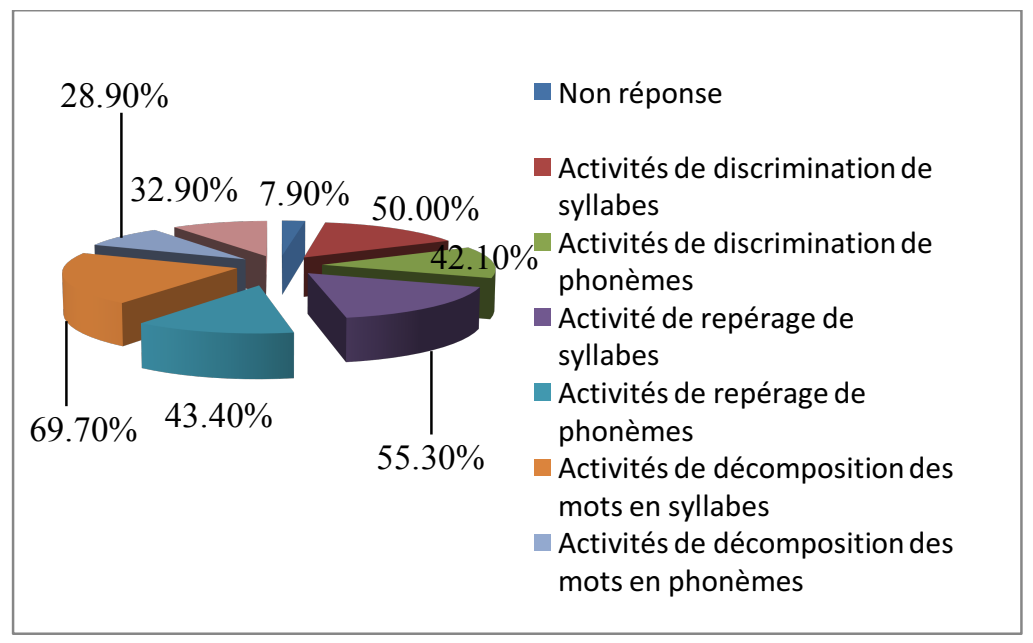

Figure 5. Activités de la conscience phonologique pratiquées selon les enseignants

\section{CONCLUSION}

Un enseignement de la lecture efficace et efficient exige que les professeurs aient des connaissances assez développées des différentes composantes de cet apprentissage ainsi que des difficultés à prévoir et des stratégies de remédiation susceptibles d'aider les élèves en difficultés à améliorer leurs performances.

Malgré les limites de notre étude, qui concernent d'une part les possibilités de généralisation des résultats à l'échelle nationale et d'autre part le fait que le questionnaire n'a pas été soutenu par des observations de la pratique enseignante de la lecture en classes ce qui aurait pu assurer plus de fiabilité à nos résultats, Cette enquête nous a permis de dresser l'état des lieux de l'enseignement apprentissage de la lecture en nous fournissant des données intéressantes sur :

- Les performances des élèves et leur niveau de maîtrise de la lecture.

- Les difficultés constatées par les praticiens.

- Les stratégies de remédiation mises en œuvre par les enseignants et leur degré d'efficacité.
En effet, Cette investigation nous a permis de déduire que les élèves de la région Taourirt-El Aioun ne font pas l'exception au Maroc en ce qui concerne la maîtrise de la lecture. Les résultats dévoilent une déficience alarmante à laquelle les stratégies de remédiation mises en œuvre par les enseignants restent peu efficaces. D'autre part, en ce qui concerne la conscience phonologique, les informateurs ont manifesté un vif besoin de formation continue à ce propos car ils jugent leurs connaissances insuffisantes.

Cette enquête nous a permis d'émettre des hypothèses à vérifier par d'autres études :

- Le développement de la conscience phonologique dès le préscolaire peut contribuer à prévenir quelques difficultés de l'apprentissage de la lecture.

- Le recours au développement de la conscience phonologique peut être un atout afin d'aider les élèves en fin du primaire à surmonter leurs difficultés de lecture.

La vérification de ces hypothèses dans le contexte marocain à travers des études longitudinales constitue un domaine de la 
recherche scientifique qui n'a pas encore été exploré, sachant que de nombreuses études menées en Europe dans différents contextes ont déjà pu confirmer la première hypothèse.

\section{REMERCIEMENTS}

Toute notre reconnaissance et notre gratitude aux responsables de la direction provinciale du ministère de l'éducation nationale de Taourirt ainsi qu'aux directeurs et enseignants des établissements scolaires ayant participé à cette enquête.

\section{RÉFÉRENCES}

Dehaene, S. (2007). Les neurones de la lecture. Paris: Odile Jacob.

Dehaene, S. (dir)., Dehaene, L.G., Gentaz. E., Huron. C. et Sprenger-Charolles. L. (2011). Apprendre à lire : des sciences cognitives à la salle de classe. Paris: Odile Jacob.

Duhazé, S.-B. (2013). Entrainement de la conscience phonologique et progrès en lecture d'élèves en grande difficulté. Paris: L'Harmattan.

Ecalle, J., et Magnan, A. (2002). L'apprentissage de la lecture : fonctionnement et développement cognitifs. Paris: Armand Colin. «U ».

Ecalle, J., et Magnan, A. (2015). L'apprentissage de la lecture et ses difficultés, Paris: Dunod. «U ».
Gillig, J-M. (2001). Remédiation, soutien et approfondissement à l'école. [S,L] : Hachette éducation. " Profession enseignant ».

Gombert, J-E. (1990). Le développement métalinguistique. Paris: PUF.

Krzywanski, N. (2013). Apprendre la conscience phonologique avec des jeux de cartes. Paris : Retz.

Melby-Lervag, M., Lyster, S.-A. et Hul, C. (2012). Phonological skills and their role in learning to read: Ametaanalytic review. Psychological Bulletin, 138 (2), 322-352.

Observatoire National de la Lecture, Inspection Générale de l'éducation nationale (France). (2005). L'apprentissage de la lecture à l'école primaire, Rapport-n ${ }^{\circ} 2005-123$.

Observatoire National de la Lecture. (1998). Apprendre à lire au cycle des apprentissages fondamentaux (GS,CP,CE1): Analyses, réflexions et propositions, ouvrage collectif dir. par Morais, J., et Robillart, G., introd. d'Alain Bentolila. Paris: Odile Jacob.

Robert, J-P. (2002). Dictionnaire pratique de didactique du FLE, Collection l'essentiel du français. [SL]: OPHRYS.

Toumi, A. (2016). L'essentiel en Didactique du Français langue étrangère: concepts, méthodologies et approches pédagogiques. Rabat: EL Maarif Al Jadida. 